\title{
A Basis Theorem for Perfect Sets
}

\author{
Marcia J. Groszek \\ Theodore A. Slaman*
}

November 17, 2000

\begin{abstract}
We show that if there is a nonconstructible real, then every perfect set has a nonconstructible element, answering a question of K. Prikry. This is a specific instance of a more general theorem giving a sufficient condition on a pair $M \subset N$ of models of set theory implying that every perfect set in $N$ has an element in $N$ which is not in $M$.
\end{abstract}

\section{Introduction}

In this paper, we consider the following question, posed by K. Prikry in the 1960's. It appeared in lists of open questions of Mathias [1979] (compiled in 1968) and Friedman [1975].

Question 1.1 (Prikry) Suppose there is a nonconstructible real. Does every perfect set have a nonconstructible element?

Rephrased, Prikry's question asks whether the assumption that there is a nonconstructible real implies that the nonconstructible reals are a basis for the perfect sets. From the opposite point of view, it asks whether it is possible for there to be a nontrivial perfect subset of $L$, Gödel's inner model of constructible sets.

*During the preparation of this paper, Slaman was partially supported by National Science Foundation Grant DMS-9500878. The authors wish to thank W. H. Woodin for the illuminating conversations they had with him on the topics of this paper. 
In private correspondence, Prikry described the environment in which he posed his question, and we paraphrase his remarks in this paragraph. Those were the early days of forcing, and his question was part of a larger question then being addressed with the tools newly available: What are the constructible reals like relative to the set of all reals? The particular import of Prikry's question is this: How substantial can the set of constructible reals be? In some models, it appears to be quite substantial. It is possible for the constructible reals to fail to be Lebesgue measurable [Solovay] or to fail to have the property of Baire [Solovay] and independently [Hrbáček and Vopěnka, 1967] (see [Jech, 1978], section 44), or even both simultaneously [Prikry], and it is possible to have only a single non-trivial degree of constructibility [Sacks, 1971]. On the other hand, Gödel [1947] suggested that the constructible reals should be fairly insubstantial relative to the set of all reals. A negative answer to Prikry's question would provide a model in which the set of constructible reals, having a perfect subset, was substantial indeed.

Prikry's problem bears on the question of the definability of the set of constructible reals. In this connection, Friedman [1975] posed the following question.

Question 1.2 (Friedman) Suppose that there is a nonconstructible real. Can the constructible reals be an uncountable $\Sigma_{1}^{1}$ set?

Friedman's question asks whether the constructible reals can be a nontrivial analytic set. By a theorem of Martin and Solovay [1970], they can be a non-trivial co-analytic set. A positive answer to Friedman's question would imply a negative answer to Prikry's question.

Velickovic and Woodin [n.d.] gave a negative answer to Friedman's question:

Theorem 1.3 (Velickovic and Woodin) Suppose that $M$ is an inner model of set theory and $\omega_{2}{ }^{M}$ is $\boldsymbol{\Sigma}_{\mathbf{1}}^{1}$. Then either $\omega_{1}^{M}$ is countable or $M$ contains all of the reals.

This suggests that Prikry's question may have a positive answer; in a similarly suggestive result in the same paper, Velickovic and Woodin show that if there is a nonconstructible real, every superperfect set of reals has a nonconstructible element. However, tending in the other direction, they also 
give an example of models $M \subset N$ for which $N$ contains a real not in $M$, but the reals of $M$ have a perfect subset in $N$.

In this paper, we answer Prikry's question positively. In Theorem 2.5, we show that if there is a nonconstructible real, then every perfect set has a nonconstructible element. Since every uncountable $\Sigma_{1}^{1}$ set has a perfect subset, this yields a strengthening of Theorem 1.3 for $L$ : if there is an uncountable $\Sigma_{1}^{1}$ subset of $L$, then every real is constructible.

We note, though without proof, that this theorem can be strengthened: If there is a nonconstructible real, then every perfect set of reals contains a perfect subset of nonconstructible reals.

Nothing here is specific to $L$. In Theorem 2.4 we give a sufficient condition on a pair $M \subset N$ of models of set theory implying that every perfect set in $N$ has an element in $N$ which is not in $M$. This leads us to similar conclusions for higher order definability classes with the appropriate large cardinal assumptions. For example, if $\omega_{1}$ is inaccessible from reals, then every uncountable $\boldsymbol{\Sigma}_{2}^{1}$ set has a perfect subset and any basis for the perfect sets is one for the uncountable $\boldsymbol{\Sigma}_{2}^{1}$ sets as well.

\section{The basis theorem}

\subsection{A technical lemma}

Definition 2.1 1 . A sequence of reals $\left\langle G_{i}: i<\omega\right\rangle$ is dense in the set $[T]$ of paths through a tree $T \subseteq{ }^{<\omega_{2}}$ if for every $\sigma$ in $T$ there is an $i$ such that $G_{i} \in[T]$ and $\sigma$ is an initial segment of $G_{i}$.

2. A path $G \in[T]$ is eventually constant in $T$ if there is an $s$ such that $G$ is either the leftmost or rightmost path in $T$ extending $G \uparrow s$.

Lemma 2.2 Let $T$ be a perfect tree, $S$ any real, and $\left\langle G_{i}: i<\omega\right\rangle$ any sequence of reals with a subset that both is dense in $[T]$ and has no element which is eventually constant in $T$. Then there are branches $X, Y \in[T]$ such that $S$ is recursive in $X, Y$ and $\left\langle G_{i}: i<\omega\right\rangle$.

We will give the proof of Lemma 2.2 in Section 2.3. 


\subsection{Proof of the basis theorem}

Definition 2.3 Suppose that $M \subseteq N$ are models of set theory. We say that ${ }^{\omega} 2^{M}$ countably covers ${ }^{\omega} 2^{N}$ if for every $H \in N$ such that $H \subset{ }^{\omega} 2^{M}$ and $H$ is countable in $N$, there is a $G \in M$ such that $H \subseteq G$ and $G$ is countable in $M$.

For example, if $\omega_{1}^{M}=\omega_{1}^{N}$ and $M \models 2^{\omega}=\omega_{1}$, then ${ }^{\omega} 2^{M}$ countably covers $\omega^{\omega} 2^{N}$ : Take a well-ordering of ${ }^{\omega} 2^{M}$ in $M$ of height $\omega_{1}^{M}$. Since $\omega_{1}^{M}=\omega_{1}^{N}$, any subset $H$ of ${ }^{\omega} 2^{M}$ which is countable within $N$ is contained in a proper initial segment $G$ of this well-ordering. Then $G \in M$ and $G$ is countable in $M$.

Theorem 2.4 Suppose that $M \subseteq N$ are models of set theory, that ${ }^{\omega} 2^{M}$ countably covers ${ }^{\omega} 2^{N}$, and that ${ }^{\omega} 2^{M} \neq{ }^{\omega} 2^{N}$. Then every perfect set in $N$ has an element which is not in $M$.

Proof: We work within $N$. For the sake of a contradiction, suppose that $T$ is a perfect tree such that $[T] \subseteq M$.

Let $S$ be a real not in $M$. Let $\vec{H}$ be any countable sequence of reals that is dense in $[T]$ and has no element which is eventually constant in $T$. Let $\vec{G}=\left\langle G_{i}: i<\omega\right\rangle$ be a sequence in $M$ whose elements contain $H$. Fix $X, Y \in[T]$ as in Lemma 2.2. By assumption, $[T] \subseteq M$ and so $X, Y \in M$; since $\vec{G} \in M$ and $S$ is recursive in $X, Y$ and $\vec{G}$, we see that $S \in M$, a contradiction.

Theorem 2.5 If ${ }^{\omega} 2 \neq{ }^{\omega} 2^{L}$, then every perfect set has a nonconstructible element.

Proof: If $\omega_{1}^{L}$ is countable, then since every perfect set is uncountable, every perfect set has an element which is not constructible. Otherwise, $\omega_{1}=\omega_{1}^{L}$, and since $2^{\omega L}=\omega_{1}^{L}$ we have ${ }^{\omega} 2^{L}$ countably covers ${ }^{\omega} 2$. By assumption, not every real is constructible, and Theorem 2.4 then implies that every perfect set has a nonconstructible element.

We can replace $L$ by any inner model $M$ satisfying $2^{\omega}=\omega_{1}$. 


\subsection{Proof of Lemma 2.2}

In order to prove Lemma 2.2, we need to produce branches $X$ and $Y$ through $T$ such that $S$ is computable from $X, Y$ and $\vec{G}$. The basic idea is this:

We define $X$ together with a sequence $\left\langle G_{x}[r]: r<\omega\right\rangle$ of elements of $\vec{G}$, so that each $G_{x}[r]$ is a path through $T$ that is not eventually constant in $T$, $X$ agrees with the $G_{x}[r]$ on longer and longer initial segments, and $S$ is coded into $X$ and $\overrightarrow{G_{x}}: S(r)$ is the value of $X$ at the first place where $X$ differs from $G_{x}[r]$. It is easy to see that $S$ will be recursive in $X$ and $\overrightarrow{G_{x}}$.

There is no problem in recursively defining such an $X$ and $\left\langle G_{x}[r]: r<\omega\right\rangle$ : Given $G_{x}[r]$ that is not eventually constant in $T$, there are infinitely many places where $G_{x}[r]$ branches left in $T$ and infinitely many places where it branches right, so we can define $X$ to agree with $G_{x}[r]$ on a long initial segment and then split off in the direction determined by $S(r)$. Then having determined that $X$ splits off from $G_{x}[r]$ through a node $\sigma$, by the density properties of $\vec{G}$, we can choose a new $G_{x}[r+1]$ extending $\sigma$. The problem is to recover $\overrightarrow{G_{x}}$ from $X, Y$ and $\vec{G}$.

To address this, we simultaneously define $Y$ and $\overrightarrow{G_{y}}$ with the same properties (except that $S$ need not be coded), in such a way that $\overrightarrow{G_{x}}$ and $\overrightarrow{G_{y}}$ can be computed from $X, Y$ and $\vec{G}$ : Given $G_{x}[r]$ and $G_{y}[r]$, we code our choice of $G_{x}[r+1]$ into $Y$ and $G_{y}[r]$ as follows. Having chosen $G_{x}[r+1]=G_{i}$, choose $n$ large enough so $G_{i}\left\lceil n \neq G_{j}\lceil n\right.$ for all $j<i$. Then let $Y$ split away from $G_{y}[r]$ (while $X$ continues to agree with $G_{x}[r+1]$ ) at some $m>n$; so $G_{x}[r+1]$ is the least $G_{i}$ such that $G_{i}$ agrees with $X$ up to the point where $Y$ splits away from $G_{y}[r]$. Then we choose $G_{y}[r+1]$, and code that choice into $X$ and $G_{x}[r+1]$ in the same way. This ensures that we can compute $\overrightarrow{G_{x}}$ and $\overrightarrow{G_{y}}$ by simultaneous recursion from $X, Y$ and $\vec{G}$, which suffices to prove the lemma.

We now present the proof of Lemma 2.2 .

Proof: Let $T$ be a perfect tree, $S$ any real, and $\left\langle G_{i}: i<\omega\right\rangle$ any sequence of reals with a subset that both is dense in $[T]$ and has no element which is eventually constant in $T$. It is safe to assume that $G_{0}$ is a path through $T$ not eventually constant in $T$.

We define $X$ and $Y$ in $[T]$ by recursion so that $S$ is recursive in $X, Y$ and $\left\langle G_{i}: i<\omega\right\rangle$. During the recursion, we use variables $G_{x}, G_{y}$, and $t$; we assign values to $G_{x}, G_{y}$ by specifying their indices $x$ and $y$ then interpreting 
$G_{x}$ and $G_{y}$ within $\left\langle G_{i}: i<\omega\right\rangle$, and we assign a value to $t$ by specifying an integer; we carry the values assigned to $G_{x}, G_{y}$, and $t$ during one step to the beginning of the next step, but these variables do not achieve a limit over the course of the recursion. When we speak of the least $G_{k}$, we are referring to the $G_{k}$ with minimal index.

Initially $X$ and $Y$ are not defined anywhere, $G_{x}=G_{y}=G_{0}$, and $t=0$.

In the recursion step, we are given that $X$ and $Y$ are defined on all numbers less than or equal to $t$ and are equal to $G_{x}$ and $G_{y}$, respectively, where defined. We now describe the $r$ th step in the recursion.

A.r.1. Let $n$ be minimal so that $n$ is greater than $t, G_{x}(n) \neq S(r)$, and $G_{x}\left\lceil n \frown\langle S(n)\rangle\right.$, the concatenation of $\left\langle G_{x}(0), \ldots, G_{x}(n-1)\right\rangle$ with $\langle S(n)\rangle$, is in $T$; we replace $G_{x}$ with the least $G_{j}$ such that $G_{j}$ extends $G_{x}\left\lceil n \frown\langle S(n)\rangle, G_{j} \in[T]\right.$, and $G_{j}$ is not eventually constant in $T$.

A.r.2. We let $s$ be the least number such that for all $i<x$, there is an $m<s$ such that $G_{i}(m) \neq G_{x}(m)$. We replace $G_{y}$ with the least $G_{k}$ so that $G_{k} \in[T], G_{k}$ is not eventually constant in $T, G_{y}$ and $G_{k}$ agree on all numbers less than or equal to $s$, and $G_{k}$ is not equal to $G_{y}$.

A.r.3. We replace $t$ with the least number $u$ such that for all $i<y$, there is an $m<u$ such that $G_{i}(m) \neq G_{y}(m)$, and extend the definitions of $X$ and $Y$ so that they agree with $G_{x}$ and $G_{y}$ on all numbers less than $u$, using the values for $G_{x}$ and $G_{y}$ assigned during steps A.r.1 and A.r.2.

This completes the definition of $X$ and $Y$.

We can now compute $S$ from $X, Y$ and $\left\langle G_{i}: i<\omega\right\rangle$ by recursion. We begin the following recursion with $r=0$ and $G_{x}=G_{y}=G_{0}$.

B.r.1. Compute $S(r)$ as the value of $X$ at the least $n$ such that $X(n)$ does not equal $G_{x}(n)$.

B.r.2. Replace $G_{x}$ with the least $G_{j}$ that agrees with $X$ on all numbers less than the first difference between $Y$ and $G_{y}$.

B.r.3. Replace $G_{y}$ with the least $G_{k}$ that agrees with $Y$ on all numbers less than the first difference between $X$ and $G_{x}$, using the value for $G_{x}$ assigned in B.r.2. 
Finally, we prove by induction on $r$ that the above computation of $S$ is correct and that, as is the case initially, the values of held by $G_{x}$ and $G_{y}$ at the beginning of step B.r equal the values held by $G_{x}$ and $G_{y}$ at the beginning of step A.r. Let $G_{x}[r]$ and $G_{y}[r]$ denote these values.

By the definition of $G_{x}$ in step A.r.1, $S(r)$ is computed correctly in step B.r.1. By the definition of $G_{y}$ in step A.r.2 and the fact that the definition of $Y$ is extended past $s$ in step A.r.3, the first number at which $Y$ and $G_{y}[r]$ disagree is larger than the value of $s$ assigned during step A.r.2, and thereby is large enough that the new $G_{x}$ computed in step B.r.2 is the one assigned to $G_{x}$ in step A.r.1. Finally, the least number at which $X$ disagrees with this new $G_{x}$ is greater than the value of $t$ set during step A.r.3. Consequently, the new $G_{y}$ of step B.r.3 is the one assigned to $G_{y}$ during step A.r.2. Thus, the new values of $G_{x}$ and $G_{y}$ at the end of step B.r are the ones held at the end of step A.r. And so, $G_{x}$ and $G_{y}$ are interpreted identically at the beginning of steps A. $r+1$ and B. $r+1$, as required.

\section{Conclusion}

We close with two questions.

Question 3.1 Does there exist a perfect tree $T$ which is not recursive such that for every element $X$ of $[T]$, if $X \leq_{T} T$ then $X$ is recursive?

Question 3.1 is the effective version of Prikry's question, replacing relative constructibility with Turing reducibility. It is related to the recursion theoretic problem of Yates: Does every minimal degree $m$ have a strong minimal cover, that is, a degree $c$ such that the only degrees less than $c$ are 0 and $m$ ? A tree such as is asked for in Question 3.1 is antithetical to a positive solution to Yates's problem.

Question 3.2 (Woodin) Assume that there is a nonconstructible subset of $\omega_{1}$. Does every countably closed perfect tree on $\omega_{1}$ have a nonconstructible path?

Our proof of Lemma 2.2 cannot be applied to Question 3.2. In Lemma 2.2 we code information into a pair of sets to be retrieved by a recursion in which the outcome of the $n$th step is needed to determine what to retrieve during the $n+1$ st step. This organization does not propagate through limit stages, which would have to be confronted in a tree on $\omega_{1}$. 


\section{References}

Friedman, H. [1975]. 102 problems in mathematical logic, J. Symbolic Logic 40: $113-129$.

Gödel, K. [1947]. What is Cantor's continuum problem?, Amer. Math. Monthly 54: 515-527.

Hrbáček, K. and Vopěnka, P. [1967]. The consistency of some theorems on real numbers, Bull. Acad. Polon. Sci. 15: 107-111.

Jech, T. [1978]. Set Theory, Academic Press.

Martin, D. and Solovay, R. [1970]. Internal Cohen extensions, J. Symbolic Logic 2: 143-178.

Mathias, A. R. D. [1979]. Surrealist landscape with figures (a survey of recent results in set theory), Period. Math. Hungar. 10: 109-175.

Sacks, G. E. [1971]. Forcing with perfect closed sets, in D. Scott (ed.), Axiomatic Set Theory I, Vol. 13 of Proc. Symp. Pure Math., American Mathematical Society, Providence, R. I., pp. 331-355.

Velickovic, B. and Woodin, W. H. [n.d.]. Complexity of the reals of inner models of set theory. unpublished. 